\title{
Discharge instructions for emergency department patients: what should we provide?
}

\author{
David McD Taylor, Peter A Cameron
}

\begin{abstract}
Effective communication between the physician and patient is required for optimum post-emergency department management. Written emergency department discharge instructions, when used to complement verbal instructions, have been shown to improve communication and patient management. This review examines the purpose, advantages, and disadvantages of three commonly used types of discharge instruction. The desirable features of discharge instructions are described. It is recommended that structured, pre-formatted instruction sheets be provided to all patients discharged to home, that emergency departments establish uniform policies to promote best practice in communication, and that the use of discharge instructions be considered as an emergency department performance indicator.

(F Accid Emerg Med 2000;17:86-90)
\end{abstract}

Keywords: discharge instructions; communication

All patients discharged to home from the emergency department should be given instructions for the ongoing management of their illness. Effective communication is the cornerstone of good medical care $\mathrm{c}^{1-3}$ and every effort should be made to ensure that discharge instructions are well understood. Unfortunately, studies have demonstrated that patient recall and understanding of diagnosis, treatment, and follow up plans are often poor..$^{2-6}$ It has been suggested that misinterpretation of instructions adversely affects compliance, correct use of medications, treatment, follow up, and outcome. ${ }^{1-3} 7-11$

Instructions may be verbal or written. ${ }^{2} 9^{11-13}$ Verbal instructions should be clear, unambiguous, to the point, and address the patient's main problems. ${ }^{2}{ }^{3} 913$ They depend upon good communication skills and an adequate doctorpatient relationship. ${ }^{31}$ It has been demonstrated that patient recall of verbal instructions can be poor, ${ }^{13}{ }^{14}$ and there is evidence that improved communication and patient management can be achieved if verbal instructions are complemented by written emergency department discharge instructions. ${ }^{2} 79$ 11-13

In this article, discharge instructions are defined as any form of documentation given to the patient or guardian, upon discharge to home, for the purpose of facilitating safe and appropriate continuity of care. Few studies have investigated the nature or quality of discharge instructions and their desirable features have not been clearly defined. This article describes three types of discharge instruction in general use and examines the advantages and disadvantages of each. Recommendations are made for the use of two types of discharge instruction, the inclusion of certain desirable features, and the direction of future research.

\section{Types of written emergency department} discharge instructions

The definition of discharge instruction is broad and discharge instructions are likely to vary widely in practice. However, three main types of may be recognised. First, an instruction note is simply a set of instructions handwritten or typed on plain paper, without the assistance of computer programs. They have the advantage of being tailored to each patient. However, they are likely to lack structure and some desirable features, may take time to prepare, and their usefulness may be limited by poor handwriting. Consequently, their adequacy may be suspect in many cases.

Second, a pre-formatted instruction sheet is a set of instructions handwritten or typed on a pre-formatted document. The text is simply completed under specific headings that are designed to be comprehensive and inclusive of all desirable features. Software now allows computer generation using minimum computer input, key words, and details of the patient attendance in the emergency department electronic log. An example of a pre-formatted instruction sheet is given in fig 1 .

Pre-formatted instruction sheets have several advantages. Simple language can be used in all pre-formatted text to improve readability and comprehension. Also, the organising of material under named headings has been shown to enhance patient recall. ${ }^{13} 1516$ Preformatted instruction sheets are tailored to the patient, may contain less physician writing but more information than unstructured instruction notes, oblige the writer to be concise, allow for quick interpretation and transfer of information to computerised patient records, and are preferred by general practitioners (GPs) ${ }^{17-22}$ Junior emergency department staff may be less likely to provide adequate discharge instruction, both verbal and written. ${ }^{4}$ The structured nature of pre-formatted instruction sheets may assist junior staff by prompting them to address all relevant components of the post-emergency department management that may otherwise go neglected. ${ }^{13}$ The main drawback of these sheets is the time required for generation. However, this can be minimised if the headings are comprehensive and the text is written in point form notation. 
Royal Hospital Accident \& Emergency Department

Discharge instruction sheet

Patient's name:

This form provides you with initial instructions about your medical care. Please keep this form and take it with you in case you need further care.

You were seen today by Drs...................................(House officer) (Specialist)

Your diagnosis...

Expected course of the illness.

Potential complications which may occur.

Instructions:

Medication prescribed (name, dose, frequency, purpose)

$1 .$.

2.

3.

Continue/change your usual medication

Return to the emergency department if

Follow up with..

Follow up within........................ days.................. weeks. at phone No

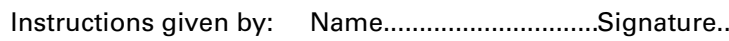

I have received and understand these instructions

Name........................................ Signature...

Figure 1 Pre-formatted instruction sheet.

Third, an information sheet is a pre-printed education and instruction document that describes one specific illness. They can be prepared for a wide array of medical illnesses or limited to several common emergency department conditions-for example, plaster or head injury instructions. Information sheets are immediately available, cheap, reproducible, and can be designed to include simple language and cartoons. However, they are not tailored to the individual patient and are disease rather than patient specific. Hence, they may not provide adequate instruction in difficult or complicated cases and may require supplementation with other discharge instructions.

\section{Purpose and advantages of discharge instructions}

PROTECTION OF THE PATIENT AND MEDICAL STAFF

The primary purpose of discharge instructions is to improve communication between physician and patient. ${ }^{13711}$ In turn, this may assist the patient with ongoing management and protect them against confusion and complications resulting from ignorance. ${ }^{7} 9112324$ Ideally, the patient will be given comprehensive discharge instructions that contain the recognised desirable features described below.

Comprehensive discharge instructions, addressing all relevant aspects of ongoing man- agement, may afford medical staff some protection from malpractice litigation. ${ }^{1}$ It is important that the hospital retains a copy of the discharge instructions, and a signed acknowledgment of their receipt, in the patient's medical record. This will provide proof that discharge instructions were provided upon discharge. If complications ensue consequent upon the patient's failure to comply with the instructions then it is less likely that the physician will be found at fault.

PATIENT AND MEDICAL STAFF EDUCATION

Some discharge instructions can serve as valuable educative material for patients. ${ }^{1} 79112324$ Although their main purpose is for the management of the present illness, they may be a useful reference in the future, especially in the event of exacerbations of chronic diseases such as asthma.

The provision of comprehensive discharge instructions can be a useful learning exercise for junior emergency department physicians. These staff must become cognisant of the natural history, potential complications, and follow up requirements of the diseases that they treat. An obligation to provide the patient with discharge instructions prompts the physician to consider and document an appropriate patient management plan and recognise the need for continuity of care between the emergency department and the patient's GP.

USE AS A GP LETTER

Comprehensive discharge instructions provide a summary of the emergency department encounter, which may also serve as a discharge letter to the patient's GP. A copy of the instructions could be forwarded to the GP using mail, facsimile, electronic transfer, or patient delivery. In this way, the GP is likely to receive the information necessary for ongoing management, communication is maintained between the emergency department and GP, and the seamless transition in patient care is facilitated.

EMERGENCY DEPARTMENT QUALITY ASSURANCE Discharge instructions can be used by the hospital and emergency department for quality assurance activities. Like other components of a patient's medical record, retained copies of discharge instructions can be examined and their adequacy assessed. This may provide information on the treatment practice of emergency department staff, and the adequacy of discharge instruction and follow up arrangements. In this way, discharge instructions may be considered as a bench mark of emergency department practice and may be used when assessing and comparing the practices of different emergency departments and their physicians.

REINFORCEMENT OF VERBAL INSTRUCTIONS

Discharge instructions must be seen as a complement to, and not a replacement for, verbal instructions. ${ }^{379121324}$ Verbal instructions are a critical component of the doctor-patient interaction where the doctor has the opportunity of 
ensuring that the patient understands the instructions and the patient has the opportunity to ask questions and clarify uncertainties. It would be a mistake to avoid this interaction and rely upon discharge instructions for all post-emergency department instruction. Furthermore, this may be perceived by the patient as impersonal and discourteous.

\section{Problems and disadvantages of discharge instructions}

The provision of verbal and written discharge instructions does not guarantee that the patient will comply with recommended postemergency department management. The patient must understand the instructions, realise their purpose, and have the motivation and means for compliance. Several studies have demonstrated that comprehension of discharge instructions may be inadequate even if they are provided..$^{2-6}$

\section{PATIENT LITERACY}

The patient must understand medical information and instructions to effectively participate in their health care. ${ }^{25122324}$ Physicians must realise that inadequate literacy may have a major impact on patient compliance and be a significant barrier to providing optimum care. ${ }^{256912}$ Even the most informative discharge instructions are of little use if they cannot be read, and therefore understood, by the patient.

Numerous studies have demonstrated that patient literacy may be limited..$^{1257122324}$ In particular, older patients are more likely to have poorer literacy. ${ }^{53}$ Studies of emergency department patient literacy have demonstrated mean reading levels ranging from grades 3 to $10.1^{12} 122324$ Furthermore, discharge instructions are often written at a standard of reading that is above the ability of many patients. ${ }^{1251224}$ The average reading level required to comprehend some discharge instructions has been shown to range from grades 6 to $14 .^{12571224}$ It has been estimated that between $45 \%$ and $50 \%$ of emergency department patients or caregivers would be unable to understand their pre-printed instructions. ${ }^{1712}$ Hence, many patients may be unable to read and understand their discharge instructions adequately. ${ }^{2571224}$ This discrepancy between patient literacy and discharge instruction readability may result in misinterpretation of instructions and poor compliance, which could adversely affect the recommended treatment and post-emergency department management. ${ }^{1} 2724$

Many authors have recommended simplification of discharge instructions in order to correct this disparity between patient literacy and instruction readability. ${ }^{125-791224}$ Simple rephrasing may improve lay person comprehension of instructions - for example, dosing of discharge medications should be stated as " $\mathrm{X}$ times a day" instead of "every X hours". ${ }^{25}$ Also, it has been demonstrated that the use of cartoons may improve the comprehension. ${ }^{9} 122627$ Patients given cartoon instructions are more likely to read instructions

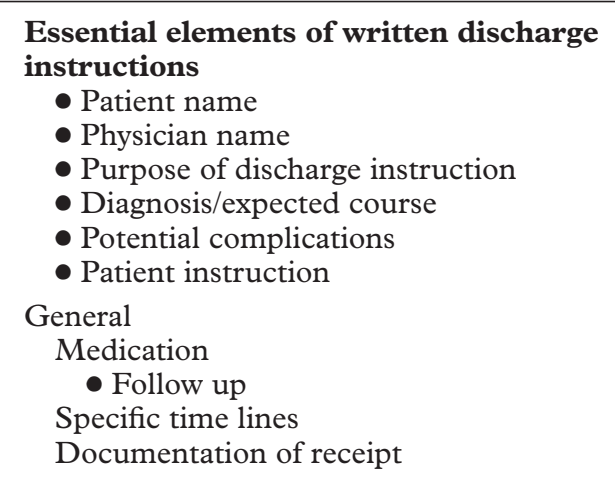

and may be more knowledgeable and compliant with post-emergency department management. ${ }^{96}$ These recommendations have been tested and revision of discharge instructions has been shown to improve patient understanding and satisfaction. ${ }^{67}$

\section{TIME OF PREPARATION}

The preparation of any documentation can be time consuming. However, the completion of pre-formatted discharge instructions, which incorporate requisite headings, prompts and check boxes, should take only about two minutes. This process may be shortened if computer programs are utilised. These allow instructions to be generated by typing in key words which results in a pertinent expanded text. For example, typing of an International Classification of Diseases diagnostic code will generate generic instructions and typing discharge drug names will generate instructions and advice on the use of those drugs.

\section{Desirable features of discharge instructions (see box)}

Little work has been done to define the necessary features of discharge instructions. The authors consider the features described below as desirable, based upon consideration of patient and medicolegal requirements, and the paucity of the literature. The incorporation of these features should provide a comprehensive and useful instruction for the patient, and perhaps the GP, but may give the impression that the discharge instructions could become cumbersome and time consuming to complete. It is noted that some features will not be applicable in many cases and do not need to be addressed. This is especially applicable to pre-formatted instruction sheets where text under inapplicable headings need not be completed.

\section{GENERAL FEATURES}

The placement of the patient's name at the top of the page should be included for specificity and may "personalise" the instructions, making them seem customised and therefore more relevant to the patient. ${ }^{11}$ Also, the name(s) of attending physicians (house officer/specialist) should be included for quality assurance. This may help to remove some of the anonymity from the episodic emergency department care from which some patients may leave without 
even noting the name of the physician who saw them. ${ }^{11}$ Also, this allows the patient the opportunity to call in to that physician(s) after discharge in the case of an unexpected complication or query. A statement of the purpose of the discharge instruction should be included to advise the patient on the proper use of the document.

ILLNESS RELATED FEATURES

The diagnosis and expected course of the illness should be included in all discharge instructions. ${ }^{11}$ This provides the patient with a realistic expectation of the natural course of the illness and may help to avoid stress from uncertainty and ignorance. Potential complications of the illness should be included. While not intended to alarm the patient, this may assist the patient in the early recognition of complications and allow prompt management. Also, at the outset, potential complications from the underlying illness can be differentiated from those of the treatment. This may help to maintain compliance with treatment regimens while minimising patient discontent and medicolegal implications.

PATIENT INSTRUCTIONS

General instructions for the management of the illness should be provided, including those that are non-drug related-for example, ice packs, rest, and elevation of an ankle sprain. The medication prescribed (name, dose, frequency, purpose) should be included and, if applicable, advice on potential complications of these drugs - for example, diarrhoea with antibiotics, abdominal discomfort after nonsteroidal anti-inflammatory drugs. ${ }^{11}$ Any alteration in the patient's usual drug regimen should be noted, for example, increase in diuretic dose. Advice on follow up is most important. ${ }^{11}$ In most cases, patients will be reviewed by their GP and an appropriate timing for review should be given, for example, seven days suture removal. ${ }^{11}$ Also, the patient should be advised when to return to the emergency department in the event of serious complications, for example, infection of laceration repair.

\section{MEDICOLEGAL FEATURES}

The name and signature of the person providing and explaining the discharge instruction should be included for quality assurance and medicolegal reasons. It has been shown that up to half of patients who have received discharge instructions were not aware that they had received them. ${ }^{14}$ Hence, a signed statement of receipt and understanding from the patient or guardian should be included. This should prompt the patient to ask for clarification of instructions, if required, and may protect the physician by documenting proof of receipt and understanding of post-emergency department management. Finally, the date and time of receipt of the instructions should be included.

\section{Recommendations}

The use of structured, pre-formatted instruction sheets for all emergency department patients discharged to home is recommended (see fig 1). A well designed instruction sheet will ensure that the desirable features described are included and considered before patient discharge. The use of accompanying information sheets are also recommended where elaboration of post-emergency department management is required-for example, plaster, head injury, gastroenteritis instructions. ${ }^{2} 791224$

The language used in these instruction sheets should be clear and simple to maximise readability. ${ }^{125-7} 9122324$ "Big" words should not be used when "small" words will suffice, for example "doctor" versus "physician". Information should be presented in clear, short sentences. Long sentences and paragraphs should be avoided whenever possible. ${ }^{59}$ Pictures and cartoons may be helpful to catch the patient's attention and if they accurately illustrate what is required of the patient. ${ }^{2591226}$ Finally, dosing of discharge medications should be stated as "number of times a day". ${ }^{25}$

Emergency departments should establish policies to ensure best practice in communication. There should be an automatic check system to ensure that discharge instructions are given to all patients upon discharge to home. The check system used will vary with the nature and resource of each emergency department but might involve computerised or emergency department chart prompts, or mandatory discharge and case management procedures. This recommendation has important quality assurance implications and consideration should be given to the use of discharge instructions as an emergency department performance indicator.

It is recommended that uniform preformatted instruction sheets be used across each health region, for example, county, state, or nation. This would aim to raise emergency department discharge instruction to best practice standards throughout the region. It would also provide a familiarity with emergency department discharge documentation for patients, emergency department staff, and GPs and allow quality assurance activities to be extended across the region.

Little work has been undertaken to describe the current discharge instruction practices of emergency departments. It is recommended that research be undertaken to describe these practices. Also, the necessary features of the instruction sheet need to be defined. This will ensure that only appropriate and relevant patient instruction is provided and that the work of discharge instruction preparation is minimised. It may be intuitive that the provision of adequate instructions improves patient management and continuity of care. Ideally, this should be demonstrated by prospective, intervention studies comparing emergency department discharge "best practice" with "usual care".

Finally, the development of computer software that facilitates the generation of discharge instructions should be continued. Well designed software should shorten the preparation time and improve the standard of discharge instructions. It could also enable the 
incorporation of the instruction directly into an electronic medical record and allow its direct electronic transfer to the patient's GP, if appropriate.

Conflict of interest: none.

Funding: none.

1 Powers RD. Emergency department patient literacy and the readability of patient-directed materials. Ann Emerg Med 1988;17:124-6.

2 Spandorfer JM, Karras DJ, Hughes LA, et al. Comprehension of discharge instructions by patients in an urban emergency department. Ann Emerg Med 1995;25:71-4.

3 Crane JA. Patient comprehension of doctor-patient communication on discharge from the emergency department. $\mathcal{F}$ Emerg Med 1997;15:1-7.

4 Logan PD, Schwab RA, Salomone JA III, et al. Patient understanding of emergency department discharge instructions. South Med $\mathcal{7}$ 1996;89:770-4.

5 Jolly BT, Scott JL, Feied CF, et al. Functional illiteracy among emergency department patients: a preliminary study. Ann Emerg Med 1993; 22: 573-8.

6 Jolly BT, Scott JL, Sanford SM. Simplification of emergency department discharge instructions improves patient comprehension. Ann Emerg Med 1995;26:443-6.

7 Chacon D, Kissoon N, Rich S. Education attainment level of caregivers versus readability level of written instructions in a pediatric emergency department. Pediatr Emerg Care 1994;10:144-9.

8 Gerson LW, Counsell SR, Fontanarosa PB, et al. Case finding for cognitive impairment in elderly emergency department patients. Ann Emerg Med 1994;23:813-17.

9 Mayeaux EJ Jr, Murphy PW, Arnold C, et al. Improving patient education for patients with low literacy skills. $A m$ Fam Physician 1996;53:205-11

10 Thomas EJ, Burstin HR, O'Neil AC, et al. Patient noncompliance with medical advice after the emergency department visit. Ann Emerg Med 1996:27:49-55.

11 Vukmir RB, Kremen R, Ellis GL, et al. Compliance with emergency department referral: the effect of computerized discharge instructions. Ann Emerg Med 1993;22:819-23.

12 Williams DM, Counselman FL, Caggiano CD. Emergency department discharge instructions and patient literacy: problem of disparity. Am ₹ Emerg Med 1996;14:19-22.
13 Isaacman DJ, Purvis K, Gyuro J, et al. Standardized instructions: do they improve communication of discharge information from the emergency department? Pediatrics 1992;89(6 pt 2):1204-8.

14 Grover G, Berkowitz CD, Lewis RJ. Parental recall after a visit to the emergency department. Clin Pediatr (Phila) 1994;33:194-201.

15 Bower GH, Clark MC, Lesgold AM, et al. Hierarchical retrieval schemes in recall of categorized word lists. Fournal of Verbal Learning and Verbal Behavior 1969;8:323-43.

16 Ley P, Bradshaw PW, Eaves D, et al. A method for increasing patients' recall of information presented by doctors. Psychol Med 1973;3:217-20.

17 Montalto M. Letters to go: general practitioners' referral letters to an accident and emergency department. Med $\mathcal{F}$ Aust 1991;155:374-7.

18 Kelly A-M. A study of the content and clarity of general practitioner referral letters to an emergency department. $N$ $Z$ Med F 1993;106:362-3.

19 Wass AR, Illingworth RN. What information do general practitioners want about accident and emergency patients? f Accid Emerg Med 1996;13:406-8.

20 Rawal J, Barnett P, Lloyd BW. Use of structured letters to improve communication between hospital doctors and general practitioners. BMF 1993;307:1044

21 Castleden WM, Stacey MC, Norman PE, et al. General practitioners' attitudes to computer-generated surgical discharge letters. Med f Aust 1992;157:380-2.

22 Jenkins S, Arroll B, Hawken S, et al. Referral letters: are form letters better? Br f Gen Pract 1997;47:107-8.

23 Jackson RH, Davis TC, Murphy P, et al. Reading deficiencies in older patients. Am f Med Sci 1994;308:7982.

24 Davis TC, Mayeaux EJ, Fredrickson D, et al. Reading ability of parents compared with reading level of pediatric patient education materials. Pediatrics 1994;93:460-8.

25 Hanchak NA, Patel MB, Berlin JA, et al. Patient misunderstanding of dosing instructions. F Gen Intern Med 1996;11: $325-8$.

26 Delp C, Jones J. Communicating information to patients: the use of cartoon illustrations to improve comprehension of instructions. Acad Emerg Med 1996;3:264-70.

27 Austin PE, Matlack R, Dunn KA, et al. Discharge instructions: do illustrations help our patients understand them? Ann Emerg Med 1995;25:317-20. 